Topical Review

\title{
Scaling Relationships Among Heart Rate, Electrocardiography Parameters, and Body Weight
}

\author{
Amanda Sarita Cruz Aleixo, DVM, MSca , Angélica Alfonso, DVM, MSc ${ }^{\mathrm{a}}$, Eunice Oba, PhD ${ }^{\mathrm{b}}$, \\ Fabiana Ferreira de Souza, $\mathrm{PhD}^{\mathrm{b}}$, Raíssa Karolliny Salgueiro Cruz, DVM, MSc ${ }^{\mathrm{a}}$, \\ Maurício Gianfrancesco Fillippi, DVM, MSc ${ }^{\mathrm{a}}$, Simone Biagio Chiacchio, $\mathrm{PhD}^{\mathrm{a}}$, \\ Miriam Tsunemi, $\mathrm{PhD}^{\mathrm{c}}$, Maria Lucia Gomes Lourenço, $\mathrm{PhD}^{\mathrm{d}, *}$
}

\section{Keywords:}

heart rate variability

dog

autonomic nervous system

temperament

Holter

allometry

${ }^{a}$ Department of Veterinary Clinics, School of Veterinary Medicine and Animal Science, UNESP, Botucatu, São Paulo, Brazil

${ }^{b}$ Department of Animal Reproduction and Veterinary Radiology, School of Veterinary Medicine and Animal Science, UNESP,

Botucatu, São Paulo, Brazil

${ }^{c}$ Department of Biostatistics, School of Biological Sciences, UNESP, Botucatu, São Paulo, Brazil

${ }^{d}$ Department of Veterinary Clinics, School of Veterinary Medicine and Animal Science, UNESP, Botucatu, São Paulo, Brazil

*Address reprint requests to Maria Lucia Gomes Lourenço, Department of Veterinary Clinics, School of Veterinary Medicine and Animal Science, UNESP, Botucatu, São Paulo, Brazil.

E-mail: mege@fmvz.unesp.br

(M.L. Gomes Lourenço)

\begin{abstract}
Although heart rate (HR) is one of the most important clinical parameters determined via physical examinations, little information is available on the normal HR in dogs, which may be related to the high variability of body weight (BW) in this species. HR is determined by the discharge rate of the sinus node, which is dependent on the autonomic nervous system and the release of catecholamines. The allometric relationship between BW and HR in different species has been described as inversely proportional; however, this relationship has been refuted. Certain authors have reported that the relationship between $\mathrm{HR}$ and BW in dogs is based on temperament as well as sympathetic autonomic stimulation of the sinus node in small breeds compared with large breeds. The aim of this study was to analyze the effects of weight, sex, age and temperament on the HR, heart rate variability and serum catecholamine (epinephrine and norepinephrine) levels in dogs. We evaluated 48 adult dogs of both sexes and various breeds and ages and divided the dogs into 5 BW groups: $<5 \mathrm{~kg}(n=8), 5-10 \mathrm{~kg}(n=10), 10-25 \mathrm{~kg}$ ( $n=10), 25-45 \mathrm{~kg}(n=10)$, and $>45 \mathrm{~kg}(n=10)$. The measured parameters were HR, breath rate (BR) and body temperature. We also performed an ambulatory electrocardiogram and electrocardiography (ECG) test for 24 hours (Holter monitor) and determined the serum levels of the catecholamines epinephrine and norepinephrine. We observed correlations between HR and sex; differences among the weight groups with respect to ECG variables and epinephrine levels; and differences among the temperament categories for certain clinical parameters, such as HR and BR. Age affected the R wave amplitude, and an allometric relationship was not observed between HR and BW in the dogs. Our results indicated that weight was associated with variations in the ECG variables; age and sex were associated with variations in HR; and temperament had a significant influence on the HR and BR of the dogs.
\end{abstract}

(c) 2017 Elsevier Inc. All rights reserved.

\section{Introduction}

In mammals, the specific metabolic rate (i.e., the metabolic rate per unit mass) decreases with increasing body size. Thus, metabolic rates are higher in small animals (such as mice) and lower in large animals (such as elephants). This inverse relationship occurs because the increased relative need for oxygen and blood flow in small animals results in significantly elevated heart rates (HRs). ${ }^{1}$

The association between metabolic rate and body weight (BW) has been widely studied, and metabolic rates are considerably higher in birds than expected based on direct proportionality alone. ${ }^{2}$ Because an animal's rate of metabolic heat production is associated with the rate at which heat is dissipated through its body surface area (BSA), BSA appears to be more appropriate for expressing the relationship between size and specific metabolic rate.,

The autonomic nervous system is defined as the peripheral motor system and subdivided into the sympathetic and

Funding for this research was provided by the Foundation for Research of the State of São Paulo-FAPESP no. 2013/10095-9. parasympathetic nervous systems, which maintain homeostasis in the body. ${ }^{5}$ The sympathetic control of the heart is mediated by adrenergic receptors, which are activated through the release of norepinephrine and epinephrine. Adrenergic receptor activation affects HRs by increasing the frequency of the pacemaker and the conduction velocity, thereby reducing the refractory period. Moreover, cardiac contractility is increased, and the overall effects are increased HR and stroke volume. ${ }^{6}$

The parasympathetic effects on the heart are mediated by the neurotransmitter acetylcholine, which activates muscarinic cholinergic receptors. Parasympathetic activation efficiently reduces cardiac pacemaker frequency and cell-to-cell conduction velocity and increases the refractory period, thereby decreasing HRs. ${ }^{7}$

The analysis of heart rate variability (HRV) enables the observation of cardiac cycle fluctuations that occur over short or long periods and the noninvasive and selective observation of autonomic function. ${ }^{8}$ The discovery of the relationship between the autonomic nervous system and cardiovascular morbidity promoted a greater number of studies on the increased sympathetic activity and reduced parasympathetic activity found in cardiovascular system diseases as well as the development of quantitative 
markers of cardiac autonomic activity, with HRV emerging as the most promising marker., ${ }^{9,10}$

Research on behavior and psychology in animals is of increasing interest because of its relevance to animal welfare. An emotion is an intense response to a short-duration event, and emotions are controlled by several different mechanisms simultaneously. Emotions are based on the activation of neural circuits in the brain that have evolved to provide greater cognitive and social assessment of the surrounding environment. ${ }^{11}$

The aim of this study was to investigate the relationship between HR and BW as well the influence of BW on clinical parameters, electrocardiography (ECG) variables ( $P$ wave, QRS complex, and $\mathrm{T}$ wave) and HRV. Additionally, we analyzed the influence of sex, age and temperament on HR, HRV and serum catecholamine (epinephrine and norepinephrine) levels in dogs.

\section{Materials and Methods}

Animals

This project was approved by the Ethics Committee on Animal Use under protocol number 41/2013-CEUA.

We evaluated 48 adult dogs of both sexes and various breeds and ages. The animals were divided into $5 \mathrm{BW}$ groups, according to the information obtained through a consultation with the American Kennel Club, which considers the following weight ranges small, medium and large. We divided the animals according to BW, aiming not to group animals of different sizes in the same group, but rather animals of similar sizes in the same group so that the size did not interfere in our results. To evaluate the influence of BW on HR: group 1: $<5 \mathrm{~kg}$ (8 animals), group 2: $5.1-10 \mathrm{~kg}(10)$, group 3: $10.1-25 \mathrm{~kg}$ (10), group 4: $25.1-45 \mathrm{~kg}$ (10) and $>45.1 \mathrm{~kg}$ (10). Information on the diet and physical activity was obtained from the owners.

The inclusion criteria for the dogs used in the study were animals that were not receiving any kind of medicine or drug and animals that, according to the owners, did not present any kind of disease. All animals that received any kind of continuous medication and animals whose health was compromised in any way were excluded from the study.

The animals used in the study presented ideal body scores according to the size of the breed. No athlete dogs or dogs that were overweight or underweight were selected to avoid compromising the results.

Allometric scaling appears to govern HR across species; accordingly, logarithmic equations have been proposed to represent the relationship between $\mathrm{HR}$ and $\mathrm{BW}$, such as $\mathrm{HR}=241 \times$ $\mathrm{BW}^{-.25} \cdot{ }^{12,13}$ We used this equation to evaluate the relationship in this study.

BSA was calculated as follows: BSA $\left(\mathrm{m}^{2}\right)=\left(10.1 \times \mathrm{BW}^{0.67}\right) \times$ $10^{-4}$, where $\mathrm{BW}$ is measured in grams.

The evaluation of temperament was performed as follows:

(1) After a period of acclimation lasting approximately 10 minutes, the demeanor of each dog was assessed via simple observations (hands-off). Dogs were scored as appearing calmrelaxed, nervous-aggressive, or excited-restless.

(2) The dogs' owners completed a questionnaire regarding the demeanor and temperament of the animals at home and in relation to animals and people who were not part of their household environment.

To classify the animals according to temperament, we used the information obtained from the questionnaires in tandem with our own observations regarding their behavior during their stay in the hospital (at first before handling and later during handling) to obtain the data for the study. These pieces of information were considered and the animals were divided in groups according to temperament.

In this study, when we mention HR Behavior, we point toward how the HR values (high HR or low HR) behave in each particular category under analysis (temperament: restless and unquiet animals tend to have higher HRs?; gender: do males or females have higher HRs; age: older animals tend to have lower HRs?) and whether there are significant differences for the variables under study. We have inserted a description of HR behavior in the study.

\section{Experimental Design}

\section{Clinical Evaluation}

After weighing, the dogs were sent along with their owners to the cardiac evaluation room.

The examination was always conducted in the same room, and the temperature was maintained between $20^{\circ} \mathrm{C}$ and $22^{\circ} \mathrm{C}$ by automatic air conditioning. HRs were counted over a period of 1 minute of cardiac auscultation and simultaneous palpation of the femoral pulse at the end of the routine physical examination (hands-on) immediately before the measurement of rectal temperature, which was performed using an electronic digital thermometer.

\section{Ambulatory Electrocardiographic Examination}

The dogs were subjected to ECG examination using a computerized $\mathrm{ECG}^{1}$ that consisted of an electronic circuit that was connected externally to a computer, which was installed with standard software. After the ECG examinations, the ECG parameters were analyzed using the software.

The animals were confined manually. Each dog was positioned in the right lateral decubitus position on a table, with the forelimbs and hindlimbs maintained at right angles to the longitudinal axis of the spine. Electrodes were placed on the skin over the elbow and stifle as standardized by. ${ }^{14}$ Three bipolar leads (I, II, and III) and 3 augmented unipolar leads (aVR, aVL, and aVF) were recorded. After the ECG recording, the results were interpreted from lead II by analyzing the following parameters: HR (bpm), electrical axis in the frontal plane (by measuring the algebraic sum of the QRS deflections in lead I and lead III) (degrees), and waves and intervals ( $\mathrm{P}$ wave $=$ duration $[\mathrm{ms}]$ and amplitude $[\mathrm{mV}] ; \mathrm{PR}$ interval [ms]; QRS = duration [ms]; R wave = amplitude [mV]; QT [ms]; polarity of T-wave [positive, negative, or biphasic]; and ST [elevation, depression, or isoelectric]).

\section{Hormonal Determination of Serum Catecholamines: Epinephrine and} Norepinephrine

To analyze the epinephrine and norepinephrine levels, blood samples $(5 \mathrm{~mL})$ were collected by venipuncture, placed in tubes for biochemical examination and centrifuged within 30 minutes after collection. Serum was aliquoted and stored at $-20^{\circ} \mathrm{C}$ until hormone determination. The concentrations of catecholamines in the serum were determined and quantified by enzyme immunoassay using the Canine Noradrenaline and Epinephrine ELISA Kit $\left(\right.$ MyBioSource $\left.^{2}\right)$. The final values after conversion were expressed in $\mathrm{pg} / \mathrm{mL}$.

\section{Dynamic Electrocardiogram Examination (Holter)}

The ECG recordings over 24 hours (Holter monitoring) were performed last and included the continuous recording of 3 ECG channels in the modified pre-cordial leads (V1, V3, and V5) using a digital apparatus (Cardio Light ${ }^{3}$ ) with an electromagnetic design 
(standard deviation). The recordings were analyzed via computerized decoding (CardioNet Client Software ${ }^{4}$ ). The recorder was directly tied to back of the animals, thus allowing freedom of movement for the dogs as well as protection for the devices. Cables were attached to adhesive electrodes placed on the skin after shaving and antisepsis according to the description of Calvert. ${ }^{15}$ After the placement of the Holter apparatus, the animals were allowed to return home. The entire monitoring session was recorded at the dogs' homes.

Indexes related to the HRV included the mean of all RR intervals (ms), standard deviation of all RR intervals ( $\mathrm{ms}$ ), average standard deviation of the measured $R R$ intervals in 5-minute segments (ms), standard deviation of RR intervals measured in 5-minute segments ( $\mathrm{ms}$ ), root mean square of successive differences in the frame between adjacent RR intervals (ms) and percentage difference between successive RR intervals $>50 \mathrm{~ms}$ (\%). The minimum, average and maximum HR were obtained using the Holter apparatus and then analyzed.

\section{Statistical Methods}

Data normality was verified using adhesion tests. Pearson (parametric test for normal distributions) and Spearman (nonparametric) correlation tests were used to test for correlations among HR, BW, and BSA in each group separately to assess whether BW is a significant factor and evaluate how other variables change as a function of BW as well as in the overall group. We conducted 5 sets of correlation analyses between BW and each of the ECG variables after 24 hours (Holter apparatus) and between BW and each of the catecholamines (norepinephrine and epinephrine).

To evaluate the effects of BW, sex, age, and temperament and their interactions on HR, HRV, and serum catecholamines, an analysis of variance was performed followed by Tukey's test.

Regression tests were performed to analyze the variables that affected HR. Linear regression models were adjusted for the dependent variables HR (obtained by different methods) by considering the following dependent variables: sex, BW, temperament and neutering.

The Kolmogorov-Smirnov test was used to test for normality. The data are presented as the mean \pm standard deviation. For all analyses, a significance level of $5 \%$ was adopted.

\section{Results}

Forty-eight dogs were evaluated and divided into 5 different BW groups. The average BW of the dogs was $23.36 \mathrm{~kg}$, and the average age was 5 years. The breeds were distributed as follows: mixed breed (22), German Shepherd (4), Poodle (3), Border Collie
(2), Lhasa Apso (2), Neapolitan Mastiff (2), Pit Bull (2), Pug (2), Australian Cattle Dog (1), Doberman (1), Golden Retriever (1), Labrador (1), Pinscher (1), Schnauzer (1), Shih Tzu (1), Yorkshire Terrier (1), and Weimaraner (1).

Regarding diet, $72.91 \%$ of the dogs were fed only dog food and $27.08 \%$ consumed both dog food and home-cooked food. Regarding temperament, 50\% (24 of 48) of the dogs were calm, 33.33\% (16 of 48 ) were agitated and $16.66 \%$ ( 8 of 48 ) were nervous. Regarding physical activity, $31.25 \%$ (15 of 48 ) presented low activity, $62.5 \%$ (30 of 48 ) presented moderate activity and $6.25 \%$ (3 of 48 ) presented high activity.

The means and standard deviations of the clinical HR, respiratory rate and body temperature were $115.58 \pm 20.39 \mathrm{bpm}, 42.08$ \pm 33.74 movements per minute $(\mathrm{mpm})$ and $38.39^{\circ} \mathrm{C} \pm .51^{\circ} \mathrm{C}$, respectively.

The means and standard deviations of the ECG parameters were as follows: P-wave duration, $53.93 \pm 7.22 \mathrm{~ms}$; amplitude, $.22 \pm .07 \mathrm{mV}$; PR interval, $101.44 \pm 18.42 \mathrm{~ms}$; QT, $192.92 \pm$ $34.37 \mathrm{~ms}$; RR, $529 \pm 130.89 \mathrm{~ms}$; QRS complex, $56.29 \pm 10.11 \mathrm{~ms}$; R-wave amplitude, $1.09 \pm .40 \mathrm{mV}$; and cardiac electrical axis, $64.00^{\circ} \pm 24.50^{\circ}$. The predominant cardiac rhythm was sinus followed by sinus arrhythmia.

As shown in Table 1, differences were observed in the BSA between each of the lower BW groups ( $<5$ and $5-10 \mathrm{~kg}$ ) and the other BW groups $(P<.0001)$. The BSA increased gradually with increasing BW. Based on the expected frequency parameter obtained using the formula $\mathrm{HR}=241 \times \mathrm{BW}^{-.25}$, the HRs differed among the groups, with smaller dogs having higher HRs.

Differences in PR interval duration were found among the $<5 \mathrm{~kg}, 10-25 \mathrm{~kg}$ and $25-45 \mathrm{~kg}$ groups $(P<.001)$, with animals in the $<5 \mathrm{~kg}$ and $5-10 \mathrm{~kg}$ groups presenting shorter PR intervals. The PR interval varied according to the HR.

Differences were found in the QRS duration $(P=.002)$ between the $<5 \mathrm{~kg}$ group and the 25-45 kg group and between the $<5 \mathrm{~kg}$ group and the $>45 \mathrm{~kg}$ group. Large dogs had longer PR interval durations and QRS complexes.

Table 3 shows the correlations obtained when the BW groups were pooled. Correlations were observed between the HR on physical examination and BSA $(P=.04 ; r=-.29)$, the HR as determined by ECG and BW $(P=.02 ; r=-.33)$, the HR determined by ECG and BSA $(P=.02 ; r=.31)$, the expected HR (HR obtained using the above-mentioned formula) and BW $(P<.0001 ; r=-.89)$, and expected HR and BSA $(P<.0001$; $r=-.93)$.

When the animals were grouped by age, differences $(P=$ .0008) were found in the R-wave amplitude between dogs younger than 5 years of age and older dogs, with older dogs exhibiting higher R-wave amplitudes $(1.30 \mathrm{mV}$ ) (younger dogs, $.92 \mathrm{mV})$.

Table 1

Clinical Parameters (Mean \pm Standard Deviation) Obtained for Dogs in the Different body weight groups

\begin{tabular}{|c|c|c|c|c|c|}
\hline Weight/Parameter & $<5 \mathrm{~kg}$ & $5.1-10 \mathrm{~kg}$ & $10.1-25 \mathrm{~kg}$ & $25.1-45 \mathrm{~kg}$ & $>45.1 \mathrm{~kg}$ \\
\hline Body weight (kg) & $3.93 \pm 0.79$ & $6.85 \pm 1.2$ & $15.46 \pm 3.53$ & $30.66 \pm 4.24$ & $56.02 \pm 10.16$ \\
\hline Age $(y)$ & $5.62 \pm 4.56$ & $5.50 \pm 3.10$ & $3.40 \pm 1.71$ & $5.70 \pm 2.54$ & $5.48 \pm 3.56$ \\
\hline HR (bpm) & $131.00 \pm 27.44$ & $122.60 \pm 19.18$ & $110.00 \pm 14.63$ & $106.60 \pm 16.49$ & $110.80 \pm 17.76$ \\
\hline $\mathrm{RR}(\mathrm{mpm})$ & $45.250 \pm 32.30$ & $62.40 \pm 61.54$ & $28.60 \pm 8.43$ & $30.80 \pm 7.72$ & $44.00 \pm 20.04$ \\
\hline$T\left({ }^{\circ} \mathrm{C}\right)$ & $38.32 \pm .81$ & $38.66 \pm .43$ & $38.55 \pm .50$ & $38.10 \pm .32$ & $38.32 \pm .34$ \\
\hline $\operatorname{BSA}\left(\mathrm{m}^{2}\right)$ & $.25 \pm .03^{\mathrm{a}}$ & $.37 \pm .04^{\mathrm{a}}$ & $.64 \pm .09^{\mathrm{b}}$ & $1.02 \pm .09^{c}$ & $1.52 \pm .18^{\mathrm{d}}$ \\
\hline $\mathrm{HR}=241 \times \mathrm{BW}(\mathrm{bpm})^{-.25}$ & $172.07 \pm 8.88^{\mathrm{a}}$ & $149.59 \pm 6.59^{\mathrm{b}}$ & $122.41 \pm 6.80^{c}$ & $102.69 \pm 3.52^{\mathrm{d}}$ & $88.48 \pm 3.86^{\mathrm{e}}$ \\
\hline
\end{tabular}

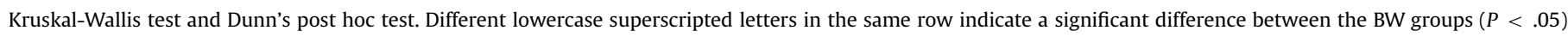
based on the Kruskal-Wallis test and Dunn's post hoc test.

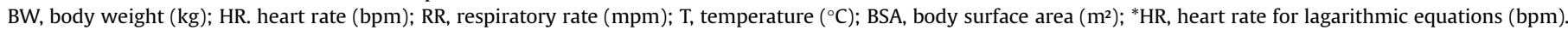

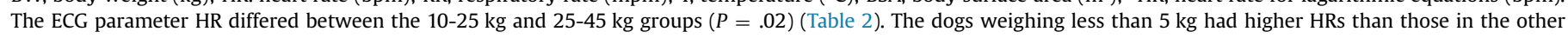
groups. 
Table 2

Electrocardiographic Parameters (Mean \pm Standard Deviation) Obtained for Dogs in the Different BW Groups

\begin{tabular}{|c|c|c|c|c|c|}
\hline Weight/Parameter & $<5 \mathrm{~kg}$ & $5.1-10 \mathrm{~kg}$ & $10.1-25 \mathrm{~kg}$ & $25.1-45 \mathrm{~kg}$ & $>45.1 \mathrm{~kg}$ \\
\hline HR (bpm) & $133.75 \pm 26.28^{\mathrm{ac}}$ & $129.70 \pm 27.05^{\mathrm{ac}}$ & $138.40 \pm 38.91^{\mathrm{ab}}$ & $103.30 \pm 21.92^{\mathrm{ac}}$ & $114.30 \pm 6.46^{\mathrm{ac}}$ \\
\hline$P(m V)$ & $.27 \pm .07$ & $.20 \pm .04$ & $.23 \pm .08$ & $.21 \pm .09$ & $.21 \pm .07$ \\
\hline $\mathrm{P}(\mathrm{ms})$ & $.04 \pm .004$ & $.05 \pm .008$ & $.05 \pm .006$ & $.05 \pm .007$ & $.05 \pm .005$ \\
\hline PR (ms) & $.08 \pm .01^{\mathrm{a}}$ & $.09 \pm .01^{\mathrm{ab}}$ & $.10 \pm .01^{\mathrm{bc}}$ & $.11 \pm .02^{\mathrm{cd}}$ & $.10 \pm .01^{\mathrm{ac}}$ \\
\hline QRS (ms) & $.04 \pm .008^{\mathrm{a}}$ & $.05 \pm .009^{\mathrm{ab}}$ & $.05 \pm .005^{\mathrm{ab}}$ & $.06 \pm .01^{\mathrm{b}}$ & $.06 \pm .008^{\mathrm{b}}$ \\
\hline QT (ms) & $.17 \pm .01$ & $.18 \pm .01$ & $.19 \pm .02$ & $.21 \pm .02$ & $.19 \pm .01$ \\
\hline $\mathrm{R}(\mathrm{mV})$ & $.96 \pm .5$ & $1.10 \pm .38$ & $1.02 \pm .34$ & $.98 \pm .37$ & $1.38 \pm .32$ \\
\hline $\mathrm{T}(\mathrm{mV})$ & $.08 \pm .24$ & $.01 \pm .30$ & $.24 \pm .08$ & $.10 \pm .33$ & $.16 \pm .24$ \\
\hline $\mathrm{RR}(\mathrm{ms})$ & $.48 \pm .12$ & $.53 \pm .14$ & $.46 \pm .13$ & $.62 \pm .13$ & $.52 \pm .06$ \\
\hline Axis $\left({ }^{\circ}\right)$ & $63.00 \pm 15.91$ & $72.30 \pm 12.45$ & $60.80 \pm 34.28$ & $53.60 \pm 35.10$ & $70.10 \pm 11.16$ \\
\hline
\end{tabular}

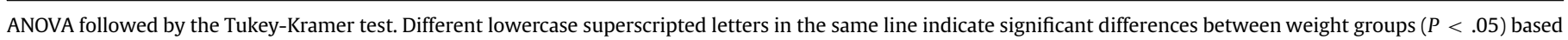
on an ANOVA followed by the Tukey-Kramer test. ANOVA, analysis of variance

Regarding the catecholamines (epinephrine and norepinephrine) evaluated in this study, differences $(P<.001)$ were found among the $10-25 \mathrm{~kg}, 25-45 \mathrm{~kg}$, and $>45 \mathrm{~kg}$ groups as well as between the $<5 \mathrm{~kg}$ and $25-45 \mathrm{~kg}$ groups and between the $<5 \mathrm{~kg}$ and $>45 \mathrm{~kg}$ groups for epinephrine (Table 4 ), with the concentration of epinephrine increasing gradually with increasing BW.

Differences were not observed between the groups regarding the HR obtained by Holter monitoring, although the mean HR was similar among the different BW groups and the value was lower than the HR obtained by the clinical examination and the allometric formula (Table 5).

When the groups were pooled, a positive correlation was found between BW and epinephrine levels $(r=.72 ; P<.0001)$ and between BSA and epinephrine levels $(r=.71 ; P<.0001)$.

Differences were found in the $\operatorname{HR}(P=.04)$ and BR $(P=.01)$ among the dogs of different temperament. Animals with a nervous temperament had the highest HR values, followed by the agitated, nervous and calm animals.

Differences in HR were found between the males (22 animals) and females $(26)(P=.03)$, with the females $(\mathrm{HR}=121 \mathrm{bpm})$ exhibiting higher HR values than the males (HR $=109 \mathrm{bpm})$. When analyzed by age group, dogs in the age group $>5$ years had highest $\mathrm{R}$ wave amplitude.

In the group of animals with BWs between 10 and $25 \mathrm{~kg}$, we observed a negative correlation $(r=-.79 ; P=.005)$ between the HR obtained by ECG and BW.

After the correlation analysis, a linear regression model was used to determine the variables that affected HR behavior. According to the model, when we analyzed the effects of sex on the HR obtained via clinical examination, the correlation coefficient was -12.77 for male dogs, and the results showed that sex had a significant influence on HR $(P=.010)$. Temperament and neutering also significantly influenced the HR behavior, and the correlation coefficient for the animals that had a calm temperament was $-11.39(P=.019)$ and for animals that had not been sterilized was $-14.36(P=.017)$.

\section{Discussion}

The BSA per unit BW increases with increasing animal size; however, the energy requirements of animals with different BWs are not related to the BW but rather to the metabolic weight. ${ }^{16}$ In the present study, we assessed the BSA in dogs in the different BW groups and found differences, with the BSA increasing with increasing dog BW.

This study showed that the dogs in the $<5 \mathrm{~kg}$ and $5.1-10 \mathrm{~kg}$ groups had higher HRs than the dogs in the other groups. This correlation can be attributed to the fact that smaller dogs have a greater metabolic rate per unit of body mass than larger dogs. Because smaller animals have a larger BSA than their mass, they produce a greater amount of heat per unit of body mass, thus requiring large energy expenditures. Therefore, small dogs are expected to have higher HR values. ${ }^{17}$

When the animals were stratified by age, older dogs were found to have higher R-wave amplitudes. Increased age is associated with increased P-wave duration, decreased Q-wave amplitude, increased R-wave amplitude and decreased HR. ${ }^{18,19}$ At young ages, sympathetic activity predominates, whereas with advancing age, the parasympathetic nervous system predominates. ${ }^{20}$ The values of the ECG parameters varied with age, thus demonstrating an effect of age.

When the dogs were divided according to sex, the females were found to have higher HRs than the males. According to our data, most of the females in our study were sterilized (randomly distributed among the groups) and most of the males had not been sterilized. The regression analysis demonstrated that males tend to have a lower HR than females. The differences between the sexes can be explained by the modulation of cell electrophysiology resulting from hormonal differences between males and females. ${ }^{20}$ The estrogen influences the modulation of the autonomic nervous system, and reduced estrogen levels cause a decrease in cardiac vagal modulation and altered autonomic cardiovascular control. $^{21}$

Table 3

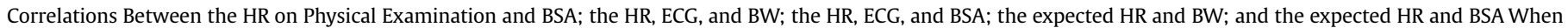
the Dogs Were Analyzed as a Single Group

\begin{tabular}{|c|c|c|c|c|c|c|}
\hline \multirow[t]{2}{*}{ Parameters } & \multicolumn{3}{|c|}{ Weight } & \multicolumn{3}{|l|}{ BSA } \\
\hline & $r$ & CI 95\% & $P$ & $r$ & CI 95\% & $P$ \\
\hline Clinical HR & -.25 & -.50 to .03 & .0830 & -.29 & -.53 to -.01 & .0421 \\
\hline HR ECG & -.33 & -.56 to -.05 & $.0212^{*}$ & -.31 & -.54 to -.03 & .0294 \\
\hline HR expected & -.89 & -.93 to -.81 & $<.0001^{*}$ & -.93 & -.96 to -.88 & $<.0001$ \\
\hline
\end{tabular}

HR, heart rate (bpm); BSA, body surface area $\left(\mathrm{m}^{2}\right)$; ECG, electrocardiogram; CI, confidence interval.

* Significant correlations $(P<.05)$. 
Table 4

Catecholamine Levels (Mean \pm Standard Deviation) Obtained for Dogs in the Different BW Groups

\begin{tabular}{|c|c|c|c|c|c|}
\hline Weight/Parameter & $<5 \mathrm{~kg}$ & $5.1-10 \mathrm{~kg}$ & $10.1-25 \mathrm{~kg}$ & $25.1-45 \mathrm{~kg}$ & $>45.1 \mathrm{~kg}$ \\
\hline Epinephrine (pg/mL) & $252.13 \pm 42.36^{\mathrm{ab}}$ & $298.50 \pm 48.79^{\mathrm{ac}}$ & $263.80 \pm 40.50^{\mathrm{ab}}$ & $349.30 \pm 79.42^{c}$ & $425.60 \pm 54.49^{d}$ \\
\hline Norepinephrine $(\mathrm{pg} / \mathrm{mL})$ & $123.00 \pm 56.40$ & $135.30 \pm 47.63$ & $120.30 \pm 33.57$ & $128.10 \pm 25.59$ & $154.60 \pm 44.64$ \\
\hline
\end{tabular}

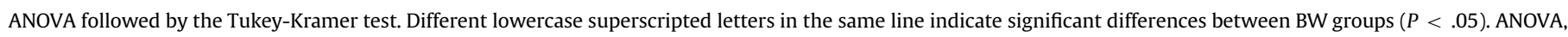
analysis of variance.

BW, body weight $(\mathrm{kg})$

In this study, animals that had not been sterilized had lower HRs, and the regression test demonstrated that neutering had a significant influence on HR behavior. In a 24-hour ECG monitoring study conducted on 51 nonsterilized dogs of different sexes, breeds, and ages, age did not differ significantly between the studied groups of dogs weighing $<20$ and $>20 \mathrm{~kg}$; moreover, the authors did not find differences in the HR between males and females, although they indicated that the anoestrous status of all females in the study had contributed to these results. ${ }^{22}$

In this study, the electrocardiographic variables (P wave duration, PR interval, QRS complex and QT interval) were influenced by BW. Differences in the regular ECG values were observed in dogs based on their body mass and included differences in the HR and $P$ wave, $R$ wave, and $T$ wave amplitude. ${ }^{22}$ In porcine species, the duration of the $\mathrm{P}$ wave, QRS complex and QT interval increase progressively as the BW of these animals increases. Similar to the dogs studied here, the referenced ECG variables in swine are also influenced by BW. ${ }^{23}$

The PR interval and the QT interval also vary according to HR, and an increase in HR and greater sympathetic stimulation decreases the duration of these variables. ${ }^{24}$

Sympathetic stimulation increases heart activity by providing rapid blood flow through the circulatory system when an individual is subjected to stress, exercise, disease or other conditions that require such an increase. ${ }^{24,25}$ The change in HR reflects the constant change of the animal's psychophysiological state, which is predominantly regulated by both branches of the autonomic nervous system. ${ }^{11}$

When the dogs were evaluated according to temperament, we observed the highest HR values in the animals with a nervous temperament, followed by the animals that were considered agitated and calm. The results of the regression test demonstrate that temperament had a significant influence on HR, with animals that were calm presenting a lower HR (coefficient $=-11.39$ ). The highest BRs were observed in the agitated animals, followed by the nervous animals and then calm animals. According to Katayama et $\mathrm{al}^{26}$ previous reports on dogs have demonstrated the influence of emotional state on HR, and the authors observed variations in the BR of dogs subjected to positive stimuli from their owners.
In this study, dogs that were considered agitated had higher epinephrine levels than the dogs with other temperaments. Increases in the secretion of plasma epinephrine from the adrenal medulla mainly occur in response to psychological stressors, ${ }^{27}$ whereas increases in the secretion of plasma norepinephrine from high numbers of sympathetic nerve endings mainly occur in response to physical stressors. ${ }^{28}$ The animals in this study were not subjected to physical stress, which likely caused the absence of correlations with norepinephrine; however, the animals may have suffered psychological stress because of the manipulation, which may have contributed to the correlations observed with epinephrine.

However, the concentration of epinephrine gradually increased according to BW, illustrating that small animals will not always present higher levels of catecholamines than large animals. Epinephrine concentrations vary according to physiology because a large animal has a higher BSA; consequently, the concentration of epinephrine will vary widely among the body regions in these animals.

Although differences were not observed among the BW groups in this study with respect to the HRV indices of the Holter examination after 24 hours (Table 5), these levels are important for evaluating autonomic functions and should be considered in the investigation of disease conditions and prognoses. ${ }^{29} \mathrm{~A}$ previous study that evaluated HR did not observe differences in dogs with different BWs after Holter monitoring ${ }^{22}$; thus, HR does not exhibit an allometric relationship with BW.

The Holter indices did not vary according to $\mathrm{BW}$, and the root mean square of successive differences in the frame between adjacent RR intervals (ms) and percentage difference between successive $R R$ intervals $>50 \mathrm{~ms}(\%)$ indices were similar among all BW groups, demonstrating that the activity of the autonomic nervous system remains the same across all BW ranges. Parasympathetic activity was the same in small and large dogs, and the mean HR was similar among all BW groups. These findings demonstrate that BW was not a determining factor for HR. The similarities found for the Holter indices among the different BW groups as well as the other parameter values obtained in this study may contribute to an HRV analysis of dogs with heart disease.

Table 5

Indexes of HRV (Mean \pm Standard Deviation) Obtained for Dogs in the Different BW Groups

\begin{tabular}{|c|c|c|c|c|c|}
\hline Weight/Parameter & $<5 \mathrm{~kg}$ & $5.1-10 \mathrm{~kg}$ & $10.1-25 \mathrm{~kg}$ & $25.1-45 \mathrm{~kg}$ & $>45.1 \mathrm{~kg}$ \\
\hline HR min & $48.12 \pm 10.23$ & $41.40 \pm 6.58$ & $44.60 \pm 19.62$ & $37.40 \pm 5.35$ & $41.80 \pm 10.78$ \\
\hline HR med & $98.87 \pm 11.67$ & $93.30 \pm 16.95$ & $83.10 \pm 12.67$ & $80.40 \pm 10.75$ & $91.10 \pm 16.45$ \\
\hline HR max & $222.50 \pm 27.81$ & $271.00 \pm 27.10$ & $271.00 \pm 27.10$ & $233.00 \pm 23.30$ & $178.50 \pm 17.85$ \\
\hline $\mathrm{NN}$ & $671.75 \pm 100.60$ & $718.90 \pm 114.33$ & $663.7 \pm 253.72$ & $801.70 \pm 125.67$ & $628.90 \pm 254.07$ \\
\hline SDNN & $245.38 \pm 57.64$ & $268.90 \pm 53.87$ & $322.30 \pm 137.79$ & $292.30 \pm 33.01$ & $294.70 \pm 130.69$ \\
\hline SDNNi & $199.75 \pm 48.94$ & $201.60 \pm 47.11$ & $260.40 \pm 137.91$ & $230.20 \pm 33.94$ & $256.50 \pm 125.93$ \\
\hline SDANN & $138.13 \pm 50.070$ & $175.60 \pm 38.17$ & $187.00 \pm 46.42$ & $173.30 \pm 66.84$ & $144.20 \pm 52.55$ \\
\hline RMSSD & $134.00 \pm 72.91$ & $142.20 \pm 54.80$ & $123.20 \pm 45.31$ & $167.30 \pm 56.01$ & $124.90 \pm 47.20$ \\
\hline pNN50 & $62.20 \pm 18.12$ & $61.22 \pm 14.01$ & $59.29 \pm 14.58$ & $59.60 \pm 9.38$ & $54.039 \pm 12.19$ \\
\hline
\end{tabular}

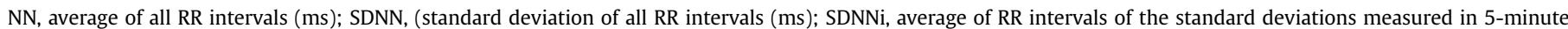

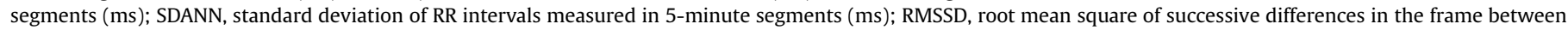
adjacent RR intervals (ms); pNN50, percentage of the difference between successive RR intervals > $50 \mathrm{~ms}(\%)$. 
We observed a correlation between the HR and BW in certain BW groups. These 2 variables exhibited a weak inversely proportional relationship, and correlations were not observed in all BW groups; thus, the intra-specific allometric relationship between HR and BW in dogs is unreliable.

The results of this study suggest that BW exerts a limited influence on HR, and autonomic nervous system activity, sex, temperament, metabolic rate and other variables have a greater influence on HR behavior than BW.

The HR values obtained using the formula $(\mathrm{HR}=241 \times$ $\mathrm{BW}^{-.25}$ ) are only estimates and yield higher HR values than those obtained using other methods (e.g., clinical measurements and ECG); thus, they should not replace conventional methods of measuring HR.

When the groups were unified, correlations were found between HR and BSA and between HR and BW; however, these correlations are considered weak. When we tested the correlations between HR values obtained using the recommended allometric formula, the results were considered strong. However, the HR values obtained using the formula were overestimated and higher than those obtained with the other methods of determining HR. These high HR values may have influenced the strong correlations found for HR obtained using the formula based on BW. Although this formula is based on an allometric scale developed in dogs, our findings indicated that an allometric relationship does not occur between HR and BW.

The present study had certain limitations. The number of animals in each group may have been insufficient to reveal certain correlations. The group of dogs between 10 and $25 \mathrm{~kg}$ included dogs with very different body sizes, which may have influenced our results for this group. Although the breed can affect certain variables, we did not standardize for breed. In addition, the vaccination protocol of the animals was not verified, which may have influenced the measured catecholamine levels.

\section{Conclusions}

Our findings indicated that analyses of HR should be performed to assess HR as a function of the animal's metabolism and HR in response to stressors, which are correlated with the animal's behavior. Moreover, our findings indicate that an allometric relationship does not occur between BW and HR in dogs.

The ECG variables were influenced by BW. Furthermore, age should be considered in HR analyses. Females have higher HR values than males, although this pattern may be related to hormonal factors.

Stress is significantly associated with changes in HR, and animals with nervous and agitated temperaments tend to have higher HRs and BRs. Environmental stimuli influence HRs and BRs, and epinephrine levels vary according to the animal's temperament and BSA. Dog behavior should be considered in future analyses of HR and HRV parameters.

\section{Authors' Contributions}

The leaders of the research group (M.L.G.L. and S.B.C.) conceived the study and the idea for the manuscript. The experiment was designed by D.A.C.Q., M.L.G.L., and S.B.C. The measurements and blood collection were performed by D.A.C.Q., M.L.G.L., C.A.D.B., and S.B.C. Endocrine analyses were performed by E.O., and biochemical analyses were performed by R.K.T. The data were analyzed D.A.C.Q., and the manuscript was written by D.A.C.Q., M.L.G.L., S.B.C., and A.A. All authors participated in the manuscript preparation and read and approved the final manuscript.

\section{Acknowledgments}

Acknowledgments to Foundation for Research of the State of São Paulo-FAPESP for funding this research.

\section{References}

1. Schwarzwald CC, Kedo M, Birkmann K, Hamlin RL. Relationship of heart rate and electrocardiographic time intervals to body mass in horses and ponies. J Vet Cardiol 14:343-350, 2012

2. Lamb AP, Meurs KM, Hamlin RL. Correlation of heart rate to body weight in apparently normal dogs. J Vet Cardiol 12:107-110, 2010

3. Ferasin L, Ferasin H, Little CJ. Lack of correlation between canine heart rate and body size in veterinary clinical practice. J Small Anim Pract 51:412-418, 2010

4. Noujaim SF, Lucca E, Muñoz V, et al. From mouse to whale: a universal scaling relation for the PR interval of the electrocardiogram of mammals. Circulation 110:2802-2808, 2004

5. Cunningham JG. Neural and hormonal control of blood pressure and blood volume. In: Cunningham JG, editor. Treaty Veterinary Physiology. 5th ed. Rio de Janeiro: Guanabara Koogan; 2014

6. Francis RCE, Pickerodt PA, Salewski L, Boemke W, Höhne C. Detection of catecholamines and metanephrines by radio-immunoassay in canine plasma. Vet J 183:228-231, 2010

7. Harada T, Ishizaki F, Hamada M, et al. Circadian rhythm of heart-rate variability and autonomic cardiovascular regulation in Parkinson's disease. Auton Neurosci 158:133-140, 2010

8. Faria EG, Nogueira SSS, Sousa MG. Assessment of heart rate variability spectral not neonatal cats and dogs. MedVep 7:354-356, 2009

9. Lopes PFF, Oliveira MIB, André SMS, et al. Clinical applicability of heart rate variability. Rev Neurosci 21:600-603, 2013

10. Rasmussen CE, Vesterholm S, Ludvigsen TP, et al. Holter monitoring in clinically healthy Cavalier King Charles Spaniels, Wire-haired Dachshunds, and Cairn Terriers. J Vet Intern Med 25:460-468, 2011

11. Zupan M, Buskas J, Altimiras J, Keeling LJ. Assessing positive emotional states in dogs using heart rate and heart rate variability. Physiol Behav 155:102-111, 2016

12. Freitas GC, Carregaro AB. Applicability of allometric extrapolation in therapeutic protocols for wildlife. Ciência Rural 43:297-304, 2013

13. Hochachka PW, Darveau CA, Andrews RD, Suarez RK. Allometric cascade: a model for resolving body mass effects on metabolism. Comp Biochem Physiol A Mol Integr Physiol 134:675-691, 2003

14. Tilley LP. Essentials of Canine and Feline Electrocardiography: Interpretation and Treatment. 3rd ed. Philadelphia: Lea \& Febiger; 1992

15. Calvert CA. Heart rate variability: advances in cardiovascular diagnostics end therapy. Vet Clin North Am Small Anim Pract 28:1409-1427, 1998

16. Parreira RP. Key issues in determining the energy requirement of domestic dogs. Rev Acad 5:415-422, 2007

17. Brito HFV. Determining the Basal metabolic rate in Dasyprocta Azarae by indirect calorimetry. [Dissertation]. Curitiba: Federal University of Paraná; 2004

18. Atmaca N, Emre B. Some electrocardiographic parameters of the Kangal dogs. J Anim Vet Adv 9:949-953, 2010

19. Wolf R, Camacho AA, Souza RCA. Computerized electrocardiography in dogs. Arq Bras Med Vet Zootec 52:317-321, 2000

20. Villareal RP, Woodruff AL, Massumi A. Gender and cardiac arrhythmias. Tex Heart Inst J 28:265-275, 2001

21. de Sá JC, Costa EC, da Silva E, Azevedo GD. Heart rate variability as a method of assessing the autonomic nervous system in polycystic ovary syndrome. Rev Bras Ginecol Obstet 35:421-426, 2013

22. Noszczyk-Nowak A, Paslawska U, Nicpoń J. ECG parameters in 24-hour holter monitoring in healthy dogs and parasitic diseases with clinic for horses, dogs, and cats. Bull Vet Inst Pulawy 53:499-502, 2009

23. Paslawska U, Noszczyk-Nowak A, Paslawski R, et al. Normal electrocardiographic and echocardiographic (M-mode and two-dimensional) values in Polish Landrace pigs. Acta Vet Scand 56:54, 2014

24. Pellegrino A, Yamaki FL, Pereira RCP, Oliveiran VMO, Larsson MHMA. Standardization of electrocardiographic parameters in Rottweiler, Golden Retriever clinically healthy. Pesq Vet Bras 30:1083-1088, 2010

25. Olsen LH, Mow T, Koch J, Pedersen HD. Heart rate variability in young, clinically healthy Dachshunds: influence of sex, mitral valve prolapse status, sampling period and time of day. J Vet Cardiol 1:7-16, 1999

26. Katayama M, Kubo T, Mogi K, Ikeda K, Nagasawa M, Kikusui T. Heart rate variability predicts the emotional state in dogs. Behav Processes 128:108-112, 2016

27. Tanno AP, Marcondes FK. Stress, reproductive cycle and cardiac sensitivity to catecholamines. Rev. Bras. Cienc. Farm 38:274-289, 2002

28. Siniscalchi M, Sasso R, Pepe AM, Dimatteo S, Vallortigara G, Quaranta A. Catecholamine plasma levels following immune stimulation with rabies vaccine in dogs selected for their paw preferences. Neurosci Lett 476:142-145, 2010

29. von Borell E, Langbein J, Després G, et al. Heart rate variability as a measure of autonomic regulation of cardiac activity for assessing stress and welfare in farm animals-a review. Physiol Behav 92:293-316, 2007 